\title{
Temperature dependent simulation of diamond depleted Schottky PIN diodes
}

Raghuraj Hathwar, Maitreya Dutta, Franz A. M. Koeck, Robert J. Nemanich, Srabanti Chowdhury, and Stephen M. Goodnick

Citation: Journal of Applied Physics 119, 225703 (2016); doi: 10.1063/1.4953385

View online: http://dx.doi.org/10.1063/1.4953385

View Table of Contents: http://aip.scitation.org/toc/jap/119/22

Published by the American Institute of Physics

\section{Articles you may be interested in}

Diamond Schottky- $p n$ diode with high forward current density and fast switching operation

Applied Physics Letters 94, 262101 (2009); 10.1063/1.3159837

Zr/oxidized diamond interface for high power Schottky diodes

Applied Physics Letters 104, 052105 (2014); 10.1063/1.4864060

Energy-band diagram configuration of $\mathrm{Al}_{2} \mathrm{O}_{3}$ /oxygen-terminated p-diamond metal-oxide-semiconductor Applied Physics Letters 107, 141601 (2015); 10.1063/1.4931123

Hydrogen-terminated diamond vertical-type metal oxide semiconductor field-effect transistors with a trench gate Applied Physics Letters 109, 033503 (2016); 10.1063/1.4958889

Thermal diffusion boron doping of single-crystal natural diamond Journal of Applied Physics 119, 205703 (2016); 10.1063/1.4949327

Charge transport properties of intrinsic layer in diamond vertical pin diode Applied Physics Letters 110, 212104 (2017); 10.1063/1.4984280

\section{AIP $\mid$ Journal of}

Save your money for your research. It's now FREE to publish with us no page, color or publication charges apply.
Publish your research in the Joumal of Applied Physics to claim your place in applied physics history. 


\title{
Temperature dependent simulation of diamond depleted Schottky PIN diodes
}

\author{
Raghuraj Hathwar, ${ }^{1}$ Maitreya Dutta, ${ }^{1}$ Franz A. M. Koeck, ${ }^{2}$ Robert J. Nemanich, ${ }^{2}$ \\ Srabanti Chowdhury, ${ }^{1}$ and Stephen M. Goodnick ${ }^{1}$ \\ ${ }^{1}$ Department of Electrical Engineering, Arizona State University, Tempe, Arizona 85287-8806, USA \\ ${ }^{2}$ Department of Physics, Arizona State University, Tempe, Arizona 85287-8806, USA
}

(Received 2 March 2016; accepted 24 May 2016; published online 8 June 2016)

\begin{abstract}
Diamond is considered as an ideal material for high field and high power devices due to its high breakdown field, high lightly doped carrier mobility, and high thermal conductivity. The modeling and simulation of diamond devices are therefore important to predict the performances of diamond based devices. In this context, we use Silvaco ${ }^{\circledR}$ Atlas, a drift-diffusion based commercial software, to model diamond based power devices. The models used in Atlas were modified to account for both variable range and nearest neighbor hopping transport in the impurity bands associated with high activation energies for boron doped and phosphorus doped diamond. The models were fit to experimentally reported resistivity data over a wide range of doping concentrations and temperatures. We compare to recent data on depleted diamond Schottky PIN diodes demonstrating low turn-on voltages and high reverse breakdown voltages, which could be useful for high power rectifying applications due to the low turn-on voltage enabling high forward current densities. Three dimensional simulations of the depleted Schottky PIN diamond devices were performed and the results are verified with experimental data at different operating temperatures Published by AIP Publishing.

[http://dx.doi.org/10.1063/1.4953385]
\end{abstract}

\section{INTRODUCTION}

Recent developments in controlling both the growth quality and doping concentration in diamond grown using plasma enhanced chemical vapor deposition (PECVD) have led to the fabrication of a variety of diamond devices such as Schottky diodes, ${ }^{1}$ p-n diodes, ${ }^{2}$ field effect transistors (FETs), ${ }^{3,4}$ and more recently bipolar junction transistors. ${ }^{5,6}$ Diamond has several desirable properties for high power electronics applications, such as a high theoretical breakdown field, ${ }^{7}$ high electron and hole mobilities at room temperature in lightly doped diamond, ${ }^{8-10}$ high thermal conductivity and extremely low intrinsic carrier concentration at room temperature. These properties lead to diamond having high potential figures of merit in both active devices, such as high-frequency field-effect transistors (FETs) and high-power switches, and passive devices such as Schottky and PIN diodes. ${ }^{11}$ The ability to accurately simulate such devices and predict the theoretical limits is therefore important. Prior attempts using Silvaco Atlas for diamond device simulations have been reported, ${ }^{12}$ but with limited success when attempting to experimental device data.

In this work, we attempt to explain the early turn-on voltage measured in p-i-n diamond diodes assuming a Schottky contact to the top P-doped n-layer which is depleted. A similar device has been reported ${ }^{13}$ and is referred to as an SPND (Schottky PN diode). The difference from an SPND device is that due to the presence of a thin lightly doped n-layer, the n-layer is completely depleted. Transport is then solely due to thermionic emission of holes making the device a unipolar device with a low turn-on voltage. 3D Atlas simulations with appropriately modified models for hopping transport in diamond were performed and fit to experimental temperature dependent I-V measurements, which verify this Schottky model for device performance.

In the following, Section II summarizes the growth and fabrication of diamond the PIN diodes compared to here. Section III discusses the simulation methodology including all the models that need to be added/modified to correctly simulate transport in $\mathrm{P}$ - and B-doped diamond materials. Section IV discusses the fitting of the simulated results to the experimental data for different devices including temperature dependent I-V data showing a good fit between theory and experiment, followed by conclusions in Section V.

\section{DIAMOND GROWTH AND DEVICE FABRICATION}

The growth and fabrication of the PIN diodes compared to here is discussed in Ref. 14. To summarize, the semiconducting layers are grown using the PECVD technique. The $\mathrm{B}$ doped layer of the diode is grown with a concentration of $\sim 10^{20} \mathrm{~cm}^{-3}$ on a $3 \mathrm{~mm} \times 3 \mathrm{~mm}$ type II-a insulating single crystal diamond (100) substrate at the Fraunhofer USA Center for Coatings and Diamond Technologies (CCD). The intrinsic layer was grown using $2 \mathrm{sccm}$ methane $\left(\mathrm{CH}_{4}\right)$ as the carbon source and $398 \mathrm{sccm}$ of hydrogen $\left(\mathrm{H}_{2}\right)$ as the carrier gas. To grow the $\mathrm{P}$ doped layer (n-layer), a gas mixture of $\mathrm{H}_{2}$ and Trimethylphosphine $\mathrm{P}\left(\mathrm{CH}_{4}\right)_{3}(200 \mathrm{ppm} / 10 \mathrm{sccm})$ is used as the $\mathrm{P}$ source. The surface is treated with an acid mixture $\left(\mathrm{HNO}_{3}: \mathrm{H}_{2} \mathrm{SO}_{4}=1: 3\right)$ to remove any surface conductive layer. Metal contacts to the $\mathrm{P}$ doped layer using $\mathrm{Ti} / \mathrm{Pt} /$ $\mathrm{Au} / \mathrm{Ni}$ with $50 \mathrm{~nm} / 50 \mathrm{~nm} / 150 \mathrm{~nm} / 50 \mathrm{~nm}$ thicknesses, respectively, were deposited using bi-layer photolithography. Square shaped contacts ranging from $50 \mu \mathrm{m} \times 50 \mu \mathrm{m}$ to $300 \mu \mathrm{m} \times 300 \mu \mathrm{m}$ and circular diodes with diameters of $50 \mu \mathrm{m}-300 \mu \mathrm{m}$ were deposited on the $\mathrm{P}$ doped layer. To reduce the impact of side-wall leakage, a single p-layer 
contact was placed at the edge of the sample as shown in Fig. 1. The B doped layer was contacted using $\mathrm{Ti} / \mathrm{Pt} / \mathrm{Au}$ with $50 \mathrm{~nm} / 50 \mathrm{~nm} / 150 \mathrm{~nm}$ thicknesses, respectively. Two approaches were explored for growing the intrinsic layers in these devices. The first approach involved growing the intrinsic layer in a chamber used for growing $\mathrm{N}$ doped diamond (Diode A). The second approach involved growing an intrinsic layer in the same chamber used for growing the $\mathrm{P}$ doped layer for both the samples (Diode B). We compare the measured I-V characteristics of these two samples with device simulations in Section IV.

\section{SIMULATION METHODOLOGY}

The simulations performed in this work use Silvaco Atlas. Atlas uses the drift-diffusion method to simulate the electrical, optical, and thermal behaviors of semiconductor devices. To properly simulate devices made from diamond, various material models had to be added and/or modified within Atlas. Our simulator incorporates the hopping transport model $^{12}$ for carriers trapped in impurity states reported by Marechal et al. Due to the high reported activation energies (several hundred $\mathrm{meV}$ ) for both $\mathrm{n}$-type ( $\mathrm{P}$ doped) and p-type (B doped) diamonds, it is essential to include an incomplete ionization model to correctly capture the free and trapped carrier concentrations. The free carrier concentrations are calculated during runtime using the charge neutrality equation,

$$
\begin{aligned}
& n+ \frac{N_{A}}{1+G V B \exp \left(\frac{E_{V}+E A B-E_{F p}}{k T}\right)} \\
&=p+\frac{N_{D}}{1+G C B \exp \left(\frac{E_{F n}-E_{C}+E D B}{k T}\right)},
\end{aligned}
$$

where $p$ and $n$ are the hole and electron free carrier concentrations, $N_{A}$ and $N_{D}$ are the acceptor and donor concentrations, $G V B=4.0$ and $G C B=0.5$ are the appropriate
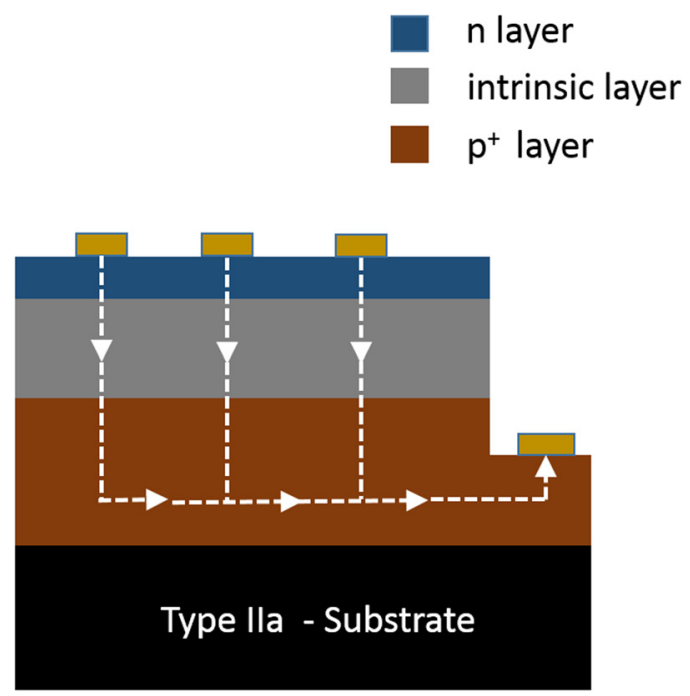

FIG. 1. Schematic representation of the device structures showing the sidewall etch contact to the $\mathrm{p}^{+}$layer and the direction of current flow. degeneracy factors for the valence band $\left(E_{V}\right)$ and conduction band $\left(E_{C}\right)$ of diamond, $E A B$ and $E D B$ are the activation energies for acceptors and donors, respectively, and $E_{F n}$ and $E_{F p}$ are the electron and hole quasi-fermi levels, respectively. In the case of $\mathrm{P}$ doped diamond, a constant value of $0.57 \mathrm{eV}$ for the activation energy is used, ${ }^{15,16}$ while for the $\mathrm{B}$ doped diamond, a significant variation in the activation energy as a function of the $\mathrm{B}$ doping concentration has been reported. ${ }^{17}$ To account for the variation in an analytical way, the activation energy as a function of doping concentration was fit to the Pearson and Bardeen model ${ }^{18}$ according to

$$
E A B(\mathrm{eV})=0.416-5.4329 \times 10^{-8} \times\left(N_{A}\right)^{\frac{1}{3}} .
$$

The parameters are chosen to get $0.362 \mathrm{eV}$ activation energy $^{19}$ at $N_{A}=1 \times 10^{18} \mathrm{~cm}^{-3}$ and zero at the metalinsulator transition ${ }^{20}$ of $4.5 \times 10^{20} \mathrm{~cm}^{-3}$. In modeling the behavior of diamond devices, another important parameter is the low-field mobility of electrons and holes. In general, they depend on the doping concentrations and the lattice temperature of the diamond. An empirical model is used which is fit to experimental Hall measurements for holes ${ }^{21}$ and for electrons. ${ }^{9}$ The empirical model is given by ${ }^{12}$ and is implemented in Atlas using the built-in C language interpreter. The temperature dependence of the band gap can also play a role at high temperatures. The model is given by ${ }^{22}$

$$
E_{g}(T)=E_{g}(300)-\frac{2 S\langle\hbar \omega\rangle}{\exp \left(\frac{\langle\hbar \omega\rangle}{k T}\right)-1},
$$

where $E_{g}(300)=5.45 \mathrm{eV}$ is the band gap at zero temperature, $S=2.31$ is a dimensionless coupling constant, and $\langle\hbar \omega\rangle=94.0 \mathrm{meV}$ is an average phonon energy.

It has been reported in homoepitaxially grown diamond that hopping conduction is prominent at high doping concentrations. In diamond with a $\mathrm{B}$ concentration between $10^{19} \mathrm{~cm}^{-3}$ and $3 \times 10^{20} \mathrm{~cm}^{-3}$, it has been reported that the conduction is of the variable range hopping (VRH) type. ${ }^{23}$ In Phosphorus doped diamond with a doping concentration between $10^{18} \mathrm{~cm}^{-3}$ and $10^{19} \mathrm{~cm}^{-3}$, the conduction is reported to be nearest neighbor hopping (NNH) conduction. ${ }^{24}$ To model devices with both n-type and p-type diamonds therefore requires an accurate model of NNH and VRH conduction. These models have been implemented in Silvaco via a built-in $C$ language interpreter. The equivalent electron mobility including $\mathrm{NNH}$ hopping mobility in $\mathrm{P}$ doped (n-type) diamond is given by

$$
\begin{gathered}
\mu_{n, e q}=\mu\left(T, N_{\text {imp }}\right)+\left(\frac{n_{h o p}}{n}\right) \mu_{h o p, N N H}, \\
\mu_{h o p, N N H}=\frac{q R_{h o p}^{2}}{6 k T} \nu_{p h} \exp \left(-2 \alpha R_{h o p}-\frac{q W_{h o p}}{k T}\right),
\end{gathered}
$$

where $\mu_{h o p, N N H}$ is the nearest neighbor hopping mobility and $n_{\text {hop }}$ is the hopping carrier concentration given by $n_{\text {hop }}$ $=N_{D}-n$ where $n$ is the free electron concentration. The localization length of the wave function $\alpha^{-1}$ is assumed to be $1.8 \mathrm{~nm}$ for donor impurities. $W_{\text {hop }}=51 \mathrm{meV}$ is the thermal activation energy for the hopping process, $R_{h o p}=N_{d}^{-1 / 3}$ is 
the mean distance between impurities, and $\nu_{p h}=2 \times$ $10^{11} \mathrm{~s}^{-1}$ is a factor related to the phonon frequency.

In Equation (5), if $2 \alpha R_{\text {hop }}$ is not negligible compared to $q W_{h o p} / k T$, the hopping probability is a maximum when $2 \alpha R_{\text {hop }}+q W_{\text {hop }} / k T$ is a minimum. This regime is called variable range hopping. On the assumption that the density of states near the Fermi energy is constant $g_{F}$, the mobility for VRH conduction in B doped (p-type) diamond is given by

$$
\begin{gathered}
\mu_{p, e q}=\mu\left(T, N_{i m p}\right)+\left(\frac{p_{h o p}}{p}\right) \mu_{h o p, V R H}, \\
\mu_{h o p, V R H}=\frac{q R_{1 / 4}^{2}}{k T} \nu_{p h} \exp \left[-\left(\frac{T_{1 / 4}}{T}\right)^{1 / 4}\right],
\end{gathered}
$$

where $\mu_{h o p, V R H}$ is the variable range hopping mobility and $p_{\text {hop }}$ is the hopping hole concentration given by $p_{\text {hop }}=N_{A}$ $-p$ where $p$ is the free hole concentration and

$$
\begin{aligned}
R_{1 / 4} & =\left(\frac{9}{8 \pi \alpha k T g_{F}}\right)^{1 / 2}, \\
T_{1 / 4} & =\frac{\beta \alpha^{3}}{g_{F} k} \\
g_{F} & =\frac{\kappa}{q^{2}}\left(\frac{3 N_{a}^{2}}{4 \pi}\right)^{1 / 3},
\end{aligned}
$$

where $\beta=7.6$ is given by $\operatorname{Mott}^{25}$ and $\kappa$ is the dielectric constant of diamond. $\alpha^{-1}$ is assumed to be $1.0 \mathrm{~nm}$ for acceptor impurities and $\nu_{p h}=2 \times 10^{13} \mathrm{~s}^{-1}$. To simulate the effect of hopping conduction, a 1D resistor with uniformly doped n-type or p-type diamond was simulated in Silvaco and the resistance is extracted from the slope of the $\mathrm{I}-\mathrm{V}$ curves for very low voltages. ${ }^{12}$ Figure 2 shows the fit between the resistivity obtained from Silvaco and the experimental data ${ }^{19}$ as a function of temperature. A compensation of $10 \%$ was used for all doping concentrations. At low temperatures, the resistivity is significantly lowered due to the presence of hopping conduction.

In Fig. 3, the range of doping concentrations where hopping conduction is dominant at room temperature is shown

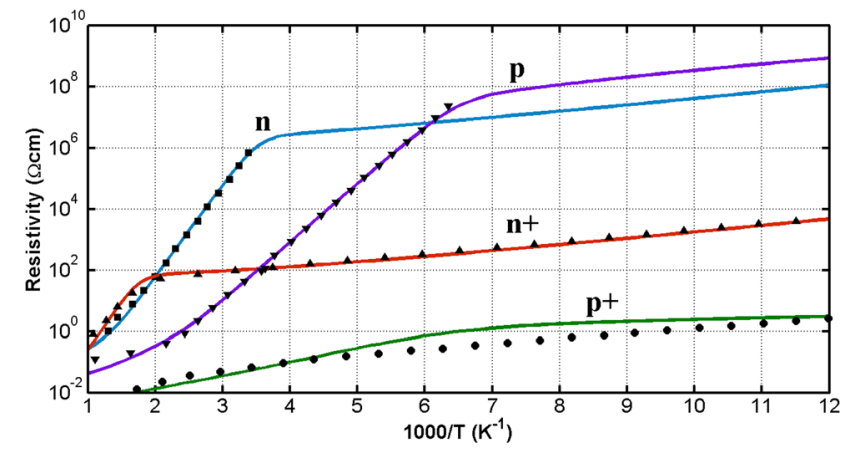

FIG. 2. Temperature dependent resistivity for B and P doped diamond films obtained from Silvaco (solid lines) plotted along with the experimental data (symbols). ${ }^{19}$ The density $(\mathrm{n})$ and $\left(\mathrm{n}^{+}\right)$represent a P concentration of $1 \times$ $10^{18} \mathrm{~cm}^{-3}$ and $8 \times 10^{19} \mathrm{~cm}^{-3}$, respectively. The density $(\mathrm{p})$ and $\left(\mathrm{p}^{+}\right)$represent a $\mathrm{B}$ concentration of $1 \times 10^{18} \mathrm{~cm}^{-3}$ and $2 \times 10^{20} \mathrm{~cm}^{-3}$, respectively.

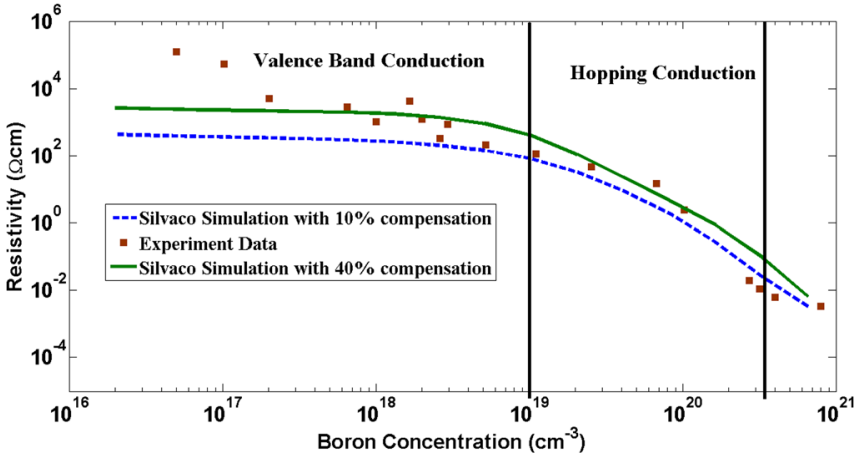

FIG. 3. Doping dependent resistivity for B doped diamond at room temperature. The solid line is with $10 \%$ compensated diamond and the dashed line is with $40 \%$ compensated diamond. Squares represent experimental data. ${ }^{17}$

in Boron doped diamond. Depending on the percentage of compensation of diamond, the resistivity can vary significantly. At very high doping $\left(\sim 3.0 \times 10^{20} \mathrm{~cm}^{-3}-4.5 \times 10^{20} \mathrm{~cm}^{-3}\right)$, the material becomes metallic in nature.

\section{SIMULATION OF DIAMOND DIODES}

Verification of the numerical models was accomplished by fitting the experimental data of diamond $\mathrm{p}$-i-n diodes ${ }^{14}$ with simulation of the same device structures in Atlas. As discussed earlier, the diodes consist of a $5.0 \mu \mathrm{m}$ thick p-type diamond with a B concentration of $\sim 10^{20} \mathrm{~cm}^{-3}$, an intrinsic layer and then a thin lightly doped n-type layer with a $\mathrm{P}$ doping concentration between $5 \times 10^{16} \mathrm{~cm}^{-3}$ and $1 \times 10^{18}$ $\mathrm{cm}^{-3}$. Using the depth profile analysis technique, ${ }^{26}$ the total thickness of the intrinsic layer and n-type layer can be determined from the $\mathrm{C}-\mathrm{V}$ data using

$$
\begin{gathered}
N(W)=\frac{2}{q \kappa A^{2} d\left(1 / C^{2}\right) / d V}, \\
W=\frac{\kappa A}{C},
\end{gathered}
$$

where $\kappa$ is the dielectric constant of diamond. The depth profile analysis technique is very useful when the doping profile is unknown or slowly varying. Unfortunately the doping profile near the surface cannot be determined this way as the capacitance required would be too large. As shown in Fig. 4, the depth at which the highly doped p-type layer present is

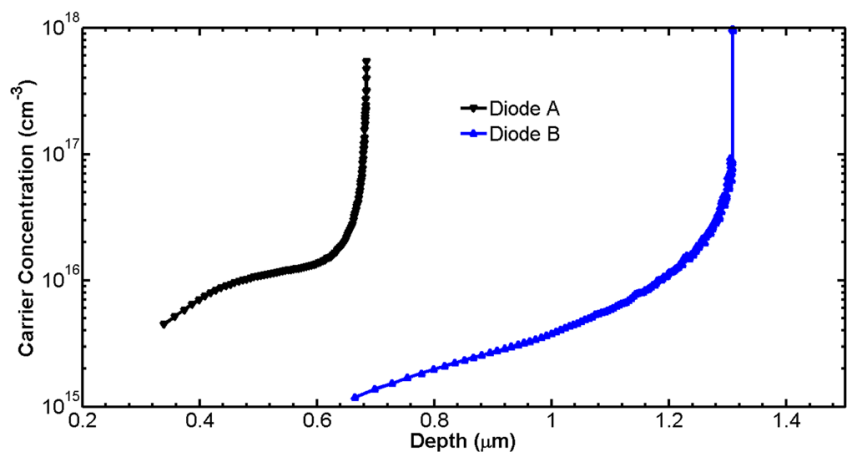

FIG. 4. Carrier concentration as a function of the depth using the depth profile analysis technique. The lower triangles represent Diode A and the upper triangles represent Diode B. 


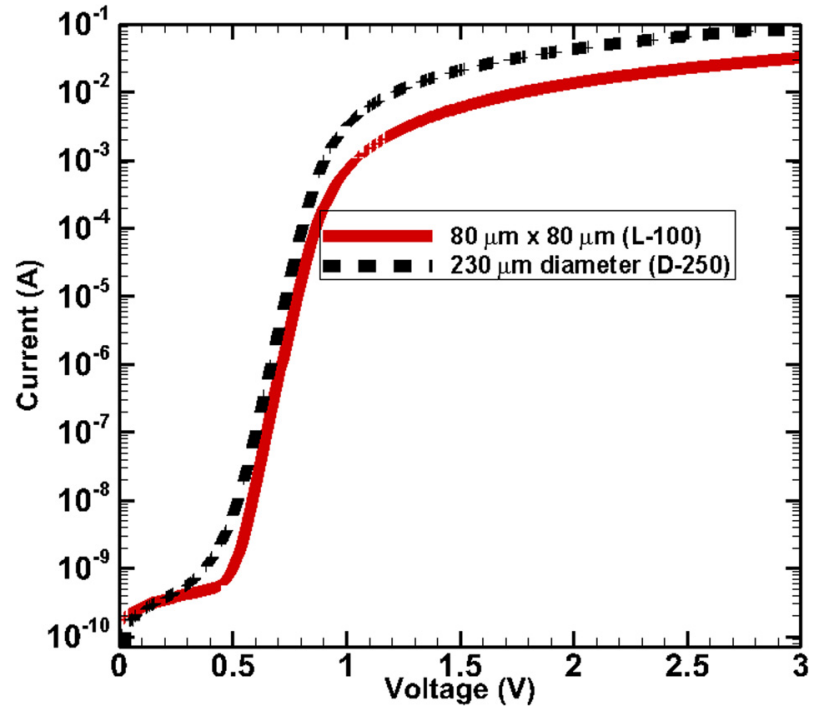

FIG. 5. I-V characteristics of the L-100 and D-250 device for Diode B. ${ }^{14}$ (b) The top view of the device showing the different sized n-layer contacts. The side wall (p-layer) contact is to the left of the figure.

around $0.65 \mu \mathrm{m}$ in Diode A and $1.3 \mu \mathrm{m}$ in Diode B. The higher carrier concentration in the intrinsic layer of Diode $\mathrm{A}$ as compared to Diode B is also shown. These results indicate that Diode A has a higher impurity concentration compared to Diode B due to the different approaches used in growing the intrinsic layer. ${ }^{14}$

The device structure consists of many small n-layer contacts and one large p-layer sidewall contact to minimize the sidewall leakage current. The current-voltage curves of two such devices from diode B is shown are Fig. 5.

To accurately simulate the series resistances, 3D simulations were performed using Silvaco incorporating the models described in Sec. III. 3D simulations were necessary since due to the side-wall contact, the transport of current in these diodes are not strictly vertical and the effect of current crowding on the series resistance near the side-wall contact may not be neglected. The side-wall contact to the p-layer extends along the whole side for $3 \mathrm{~mm}$. The top n-layer contacts are of different sizes depending on the device. The player contact is on the side as shown in Fig. 1. The thickness of the n-layer was assumed to be $50 \mathrm{~nm}$ thick with a peak Phosphorus doping concentration of $10^{17} \mathrm{~cm}^{-3}$ at the surface.

Figure 6(a) shows the fit between experimental data and Silvaco simulations at room temperature. A Schottky contact at the n-layer with barrier height of $1.27 \mathrm{eV}$ was found to provide a good fit to the early turn on voltage of the device. The formation of a Schottky barrier diode is attributed to the low $\mathrm{P}$ doping concentration and thinness of the n-layer ${ }^{13}$ which causes the entire n-doped layer to be depleted. The n-layer causes a small band bending as shown in Fig. 6(b), but due to its low doping and small thickness, it is completely depleted. This is similar to the SPND device reported by Makino et al. ${ }^{13}$ except that in the SPND device, there is a thick n-layer which causes the device to be bipolar and have a greater than $4.0 \mathrm{~V}$ turn on voltage. A shunt resistance of $\mathrm{R}_{\mathrm{sh}}=4 \times 10^{8} \Omega$ was added to account for the side-wall leakage and other leakage paths and to reproduce the low voltage resistance, before the diode turns on. An ideality factor of 1.08 was obtained, which is very close to the ideal value signifying a very smooth Schottky contact. ${ }^{27}$ A background trap density of $10^{15} \mathrm{~cm}^{-3}$ in the intrinsic layer at an energy level of $0.34 \mathrm{eV}$ above the valence band was included in the simulation to account for impurities during the growth process.

For diode B, the temperature dependent IV curves obtained experimentally for the L-400 device are shown in Fig. 7.

In an ideal Schottky diode, the current is given by

$$
I=S A^{*} T^{2} e^{-\frac{q \phi_{b}}{k T}\left(e^{\frac{q V}{k T}}-1\right),}
$$

where $S$ is the surface area of the contact, $A^{*}$ is the Richardson constant, $T$ is the temperature, $\phi_{b}$ is the barrier height of the Schottky contact, and $V$ is the applied voltage. According to Equation (8), a plot of $\ln \left(I / T^{2}\right)$ vs $(1 / k T)$ should be a straight line with a slope given by

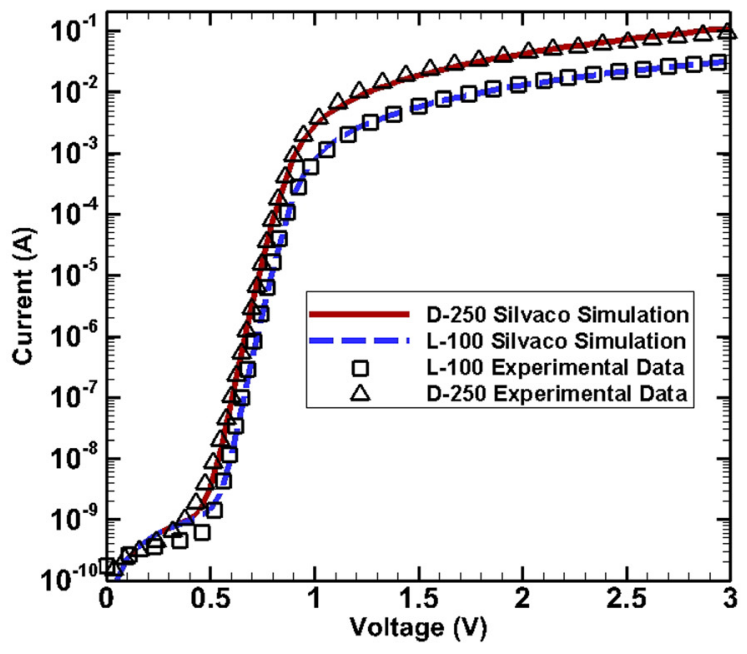

(a)

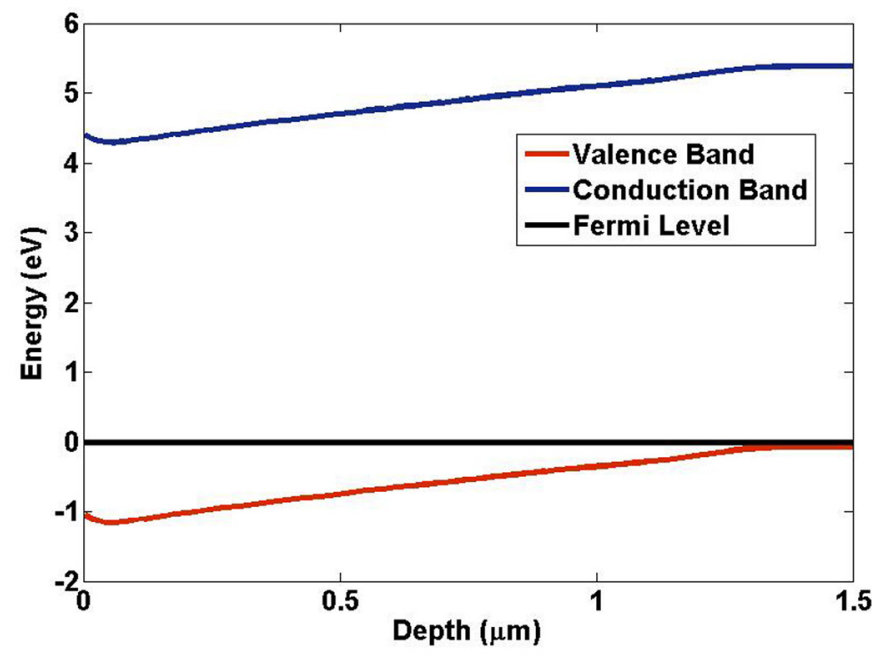

(b)

FIG. 6. (a) Comparison between the simulated I-V curves and experimental I-V curves for D-250 and L-100 devices of Diode B. ${ }^{14}$ (b) the band diagram at equilibrium for Diode B. 


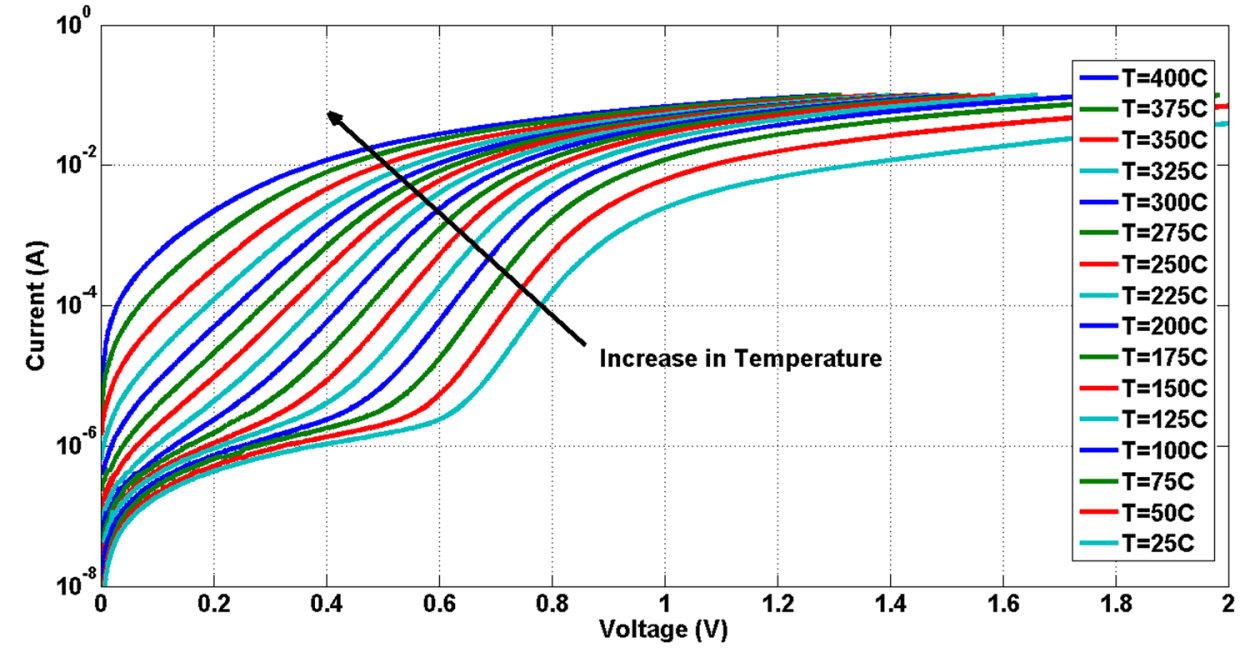

FIG. 7. Temperature dependent current voltage characteristics measured for the L-400 device of Diode B. The temperature is increased from $25^{\circ} \mathrm{C}$ to $400{ }^{\circ} \mathrm{C}$ in steps of $25^{\circ} \mathrm{C}^{30}$

$$
\text { slope }=-q\left(\phi_{b}-V\right) \text {. }
$$

The Richardson plot and the corresponding barrier height calculated using Eq. (8) is shown in Figs. 8(a) and 8(b). The straight line in Fig. 8(a) indicates the diode current is limited by thermionic emission as shown in Equations (8) and (9). This agrees with the Silvaco simulation of Diode A.

The variation of the barrier height with voltage also signifies the deviation from ideal Schottky diode behavior. This leads to an ideality factor $n>1 .{ }^{27}$ If the barrier height $\phi_{b}$ depends on voltage, then based on Eq. (8), the ideality factor $n$ is given by

$$
n=\frac{\partial V}{\partial \ln (I)}=\frac{1}{1-\partial \phi_{b} / \partial V} .
$$

Therefore, a positive variation in barrier height with voltage will lead to a larger than 1.0 ideality factor according to Equation (10). The reason behind the variation in barrier height with voltage has been widely studied. ${ }^{27-29}$ Tung's model $^{27}$ attributes barrier height inhomogeneities to the non-ideal behavior of Schottky diodes. When a region of low barrier height is surrounded by high barrier regions, the low barrier region can become "pinched" off by the high barrier region. This pinch off causes the effective barrier height to vary with voltage as well as temperature depending on the nature of the low barrier regions. Using Silvaco, 3D simulations of the L-400 device in Diode A were simulated and fit to the experimental data. Figure 9 shows the experimental and simulated data at different temperatures.

A shunt resistance of $1.6 \times 10^{5} \Omega$ was added to the simulation to account for the side-wall leakage. In order to obtain the fit, the barrier height had to be varied with voltage and temperature as shown in Table I. Similar trends in barrier height with voltage and temperature is predicted by Tung's model ${ }^{27}$ and is observed in a variety of Schottky devices.

The reverse characteristics of the devices in Diode A and Diode B are shown in Fig. 10. The inset of Fig. 10 shows the forward characteristics of the devices. The cross-section of the device shown in Diode A is a circular diode of diameter equal to $113 \mu \mathrm{m}$ and the device shown as Diode B is a square diode of side $150 \mu \mathrm{m}$. Due to the Schottky contact at the $\mathrm{P}$ doped layer, the leakage current is higher than that from a PIN device. Nevertheless, the diodes show rectification behavior with an on to off ratio of over $10^{7}$ at $4 \mathrm{~V} .{ }^{14}$ Recent developments in the growth of the $\mathrm{P}$ doped layer and

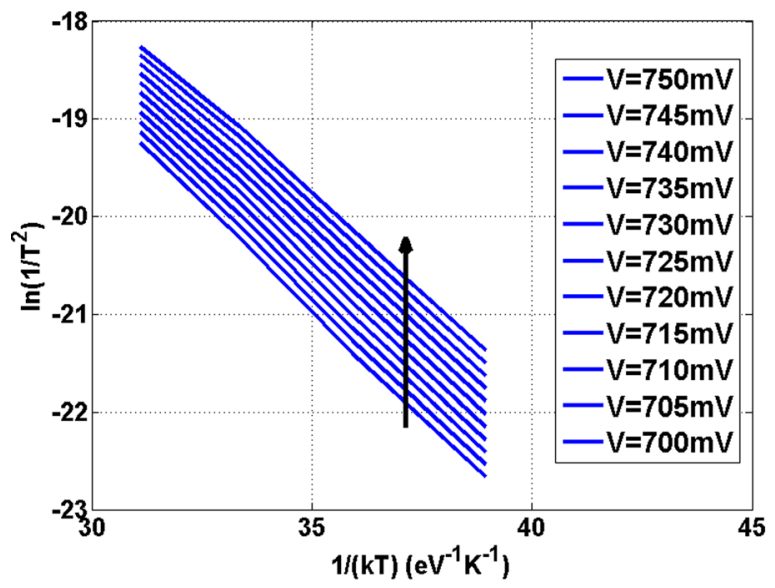

(a)

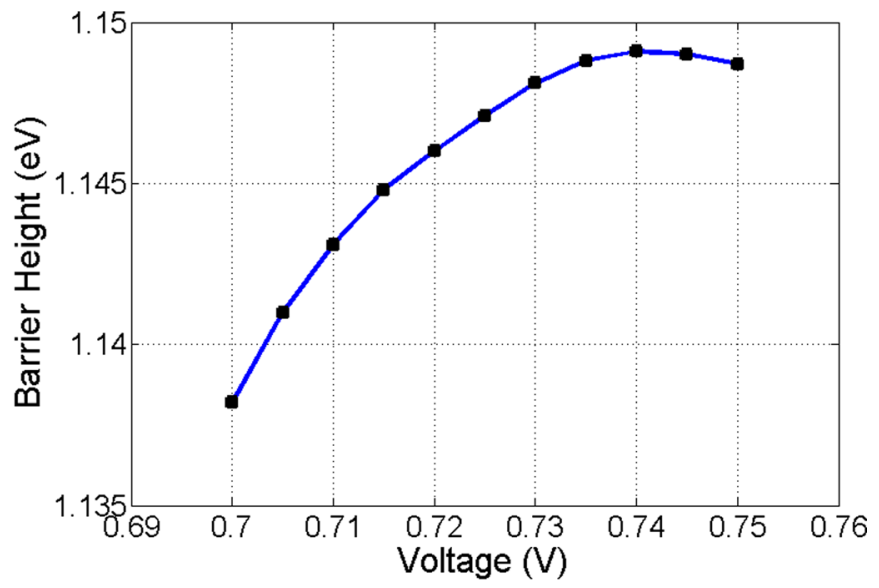

(b)

FIG. 8. (a) Richardson plot of the experimental data ${ }^{30}$ given in Fig. 7 showing the straight lines confirming the presence of a Schottky contact. (b) The variation of barrier height with voltage using Eq. (10). 


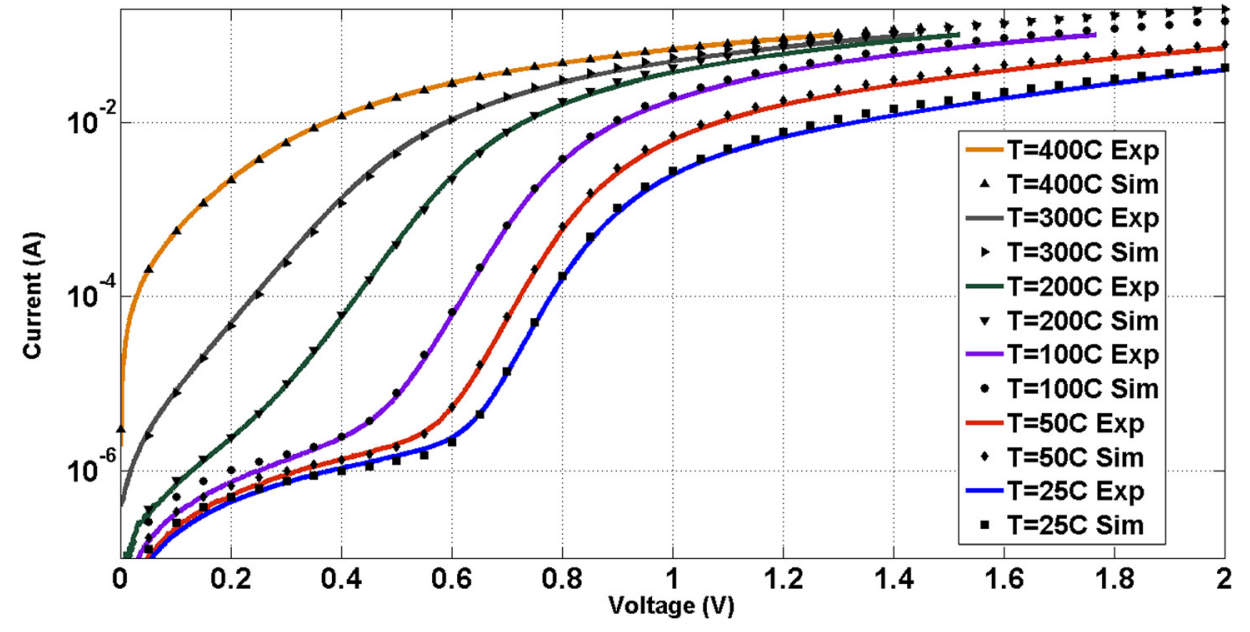

FIG. 9. Temperature dependent I-V curves of simulated data using Silvaco (solid lines) compared to the I-V curves of experimental data ${ }^{30}$ (symbols) for the L-400 device of Diode A.
TABLE I. Change in ideality factor and barrier height with temperature used in the Silvaco simulations.

\begin{tabular}{lcc}
\hline \hline Temperature $(\mathrm{K})$ & Barrier height $(\mathrm{eV})$ & Ideality factor $(\mathrm{n})$ \\
\hline 273 & 1.059 & 1.3 \\
323 & 1.085 & 1.25 \\
373 & 1.098 & 1.25 \\
473 & 1.112 & 1.23 \\
573 & 1.139 & 1.17 \\
673 & 1.053 & 1.20 \\
\hline \hline
\end{tabular}

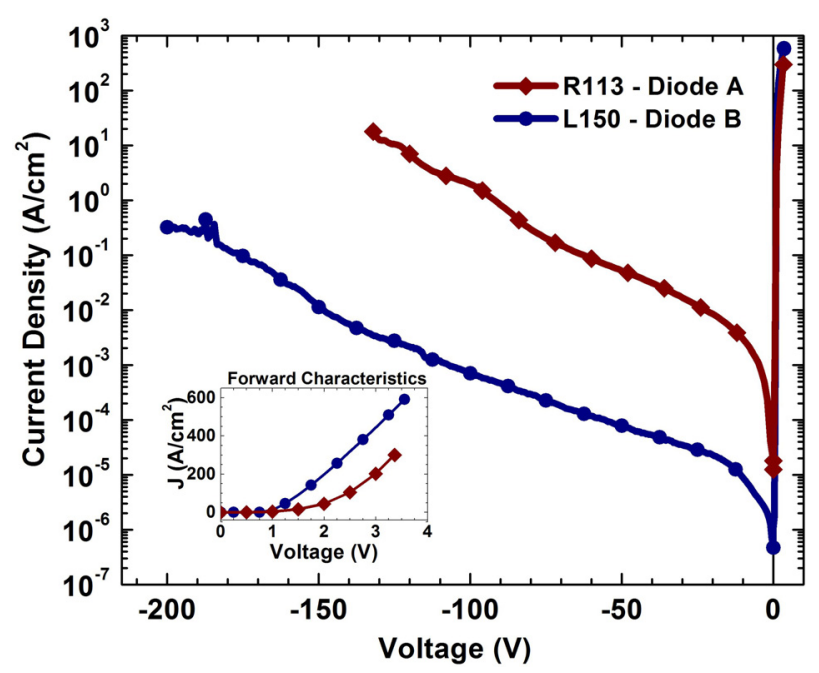

FIG. 10. Reverse current characteristics of Diode A and Diode B showing the leakage currents. The inset shows the forward current characteristics of the same devices. The contact area on the P doped layer was used to calculate the current density. Device R113 is a circular contact with radius $113 \mu \mathrm{m}$ from Diode A and device L150 is a square contact with side $150 \mu \mathrm{m}$ from Diode B.

using a thicker intrinsic layer have reduced the reverse leakage of these diodes and devices with breakdown voltages $>600 \mathrm{~V}$ reported. ${ }^{30}$

There are several advantages to a diamond depleted Schottky PIN diode over both the Schottky p-diamond diode and the bipolar diamond diodes. Due to the unipolar nature of the depleted Schottky PIN diode, the ideality factor is close to 1.0 which is advantageous in switching applications over the bipolar device in which the ideality factor is limited by recombination. Also, having a low turn-on voltage leads to higher forward current densities at low voltages. The diamond depleted Schottky PIN diodes have been reported to have a greater than $100 \mathrm{~A} / \mathrm{cm}^{2}$ current density at a forward voltage of $4.0 \mathrm{~V}$ (Ref. 14) which is higher than any reported bipolar diamond diode. Also, having an intrinsic layer leads to a high breakdown voltage which is advantageous over a simple Schottky p-diamond diode. A reverse breakdown field of greater than $600 \mathrm{~V}$ has been reported in a diamond depleted Schottky PIN diode. ${ }^{30}$ Having a low turn-on voltage while maintaining a high reverse breakdown field is desired in high power rectifying applications to minimize the power loss in the forward direction.

\section{CONCLUSIONS}

Recent developments in the fabrication of depleted Schottky PIN diodes show a high forward current density at low voltages due to an early turn-on voltage and high reverse breakdown voltages. These are desirable properties to have in high power rectifying applications. In this work, 3D Silvaco simulations of depleted Schottky PIN diamond diodes have been accurately fit to experimental data for the first time by the addition of models such as hopping conduction and partial ionization of impurities. The hopping conduction, low-field hall mobility, and other temperature dependent models have been extracted from experimental data. Temperature analysis of the diamond diodes has shown it to be Schottky in nature due to the low doping concentration of the n-layer and Silvaco simulations have verified this claim. Variations in barrier height and ideality factor with temperature and voltage have been explained using Tung's fluctuating barrier model.

\section{ACKNOWLEDGMENTS}

This research was supported by the ARPA-E SWITCHES Program under Grant No. "DE-AR0000453."

${ }^{1}$ P.-N. Volpe, P. Muret, J. Pernot, F. Omnes, T. Teraji, Y. Koide, F. Jornard, D. Planson, P. Brosselard, N. Dheilly, B. Vergne, and S. Scharnholz, Appl. Phys. Lett. 97, 223501 (2010). 
${ }^{2}$ Y. Hoshino, H. Kato, T. Makino, M. Ogura, T. Iwasaki, M. Hatano, and S. Yamasaki, Phys. Status Solidi A 209(9), 1761-1764 (2012).

${ }^{3}$ S. A. O. Russell, S. Sharabi, A. Tallaire, and D. A. J. Moran, IEEE Electron Device Lett. 33, 1471 (2012).

${ }^{4}$ T. Iwasaki, Y. Hoshino, K. Tsuzuki, H. Kato, T. Makino, M. Ogura, D. Takeuchi, T. Matsumoto, H. Okushi, S. Yamasaki, and M. Hatano, Appl. Phys. Express 5, 091301 (2012).

${ }^{5}$ H. Kato, K. Oyama, T. Makino, M. Ogura, D. Takeuchi, and S. Yamasaki, Diamond Relat. Mater. 27-28, 19-22 (2012).

${ }^{6}$ H. Kato, T. Makino, M. Ogura, D. Takeuchi, and S. Yamasaki, Diamond Relat. Mater. 34, 41-44 (2013).

${ }^{7}$ S. Koizumi, C. E. Nebel, and M. Nesladek, Physics and Applications of CVD Diamond (Wiley-VCH, 2008).

${ }^{8}$ J. Pernot, C. Tavares, E. Gheeraert, E. Bustarret, M. Katagiri, and S. Koizumi, Appl. Phys. Lett. 89, 122111 (2006).

${ }^{9}$ J. Pernot and S. Koizumi, Appl. Phys. Lett. 93, 052105 (2008).

${ }^{10}$ J. Pernot, P. N. Volpe, F. Omnes, P. Muret, V. Mortet, K. Haenen, and T. Teraji, Phys. Rev. B 81, 205203 (2010).

${ }^{11}$ C. J. H. Wort and R. S. Balmer, Mater. Today 11, 22 (2008).

${ }^{12}$ A. Marechal, N. Rouger, J.-C. Crebier, J. Pernot, S. Koizumi, T. Teraji, and E. Gheeraert, Diamond Relat. Materi. 43, 34-42 (2014).

${ }^{13}$ T. Makino, K. Oyama, H. Kato, D. Takeuchi, M. Ogura, H. Okushi, and S. Yamasaki, Jpn. J. Appl. Phys. 53, 05FA12 (2014).

${ }^{14}$ M. Dutta, F. A. M. Koeck, R. J. Nemanich, and S. Chowdhury, in Device Research Conference (DRC) (2015), p. 184.

${ }^{15}$ S. Koizumi, T. Teraji, and H. Kanda, Diamond Relat. Mater. 9, 935 (2000).

${ }^{16}$ E. Gheeraaert, S. Koizumi, T. Teraji, H. Kanda, and M. Nesladek, Diamond Relat. Mater. 9, 948 (2000).
${ }^{17}$ J.-P. Lagrange, A. Deneuville, and E. Gheeraert, Diamond Relat. Mater. 7, 1390-1393 (1998).

${ }^{18}$ G. L. Pearson and J. Bardeen, Phys. Rev. 75, 865 (1949).

${ }^{19}$ K. Oyama, S.-G. Ri, H. Kato, M. Ogura, T. Makino, D. Takeuchi, N. Tokuda, H. Okushi, and S. Yamasaki, Appl. Phys. Lett. 94, 152109 (2009).

${ }^{20}$ T. Klein, P. Achatz, J. Kacmarcik, C. Marcenat, F. Gustafsson, J. Marcus, E. Bustarret, J. Pernot, F. Omnes, B. E. Sernelius, C. Persson, A. F. Silva, and C. Cytermann, Phys. Rev. B 75, 165313 (2007).

${ }^{21}$ P.-N. Volpe, J. Pernot, P. Muret, and F. Omnes, Appl. Phys. Lett. 94, 092102 (2009).

${ }^{22}$ K. P. O’Donnell and X. Chen, Appl. Phys. Lett. 58, 2924 (1991).

${ }^{23}$ T. Sato, K. Ohashi, H. Sugai, T. Sumi, K. Haruna, H. Maeta, N. Matsumoto, and H. Otsuka, Phys. Rev. B 61, 12970 (2000).

${ }^{24}$ T. Matsumoto, H. Kato, T. Makino, M. Ogura, D. Takeuchi, H. Okushi, and S. Yamasaki, Jpn. J. Appl. Phys. 53, 05FP05 (2014).

${ }^{25}$ N. F. Mott, Conduction in Non-Crystalline Materials (Clarendon, Oxford, 1993), p. 32.

${ }^{26}$ D. K. Schroder, Semiconductor Material and Device Characterization, 2nd ed. (John Wiley \& Sons, Inc., New York, NY, 1998), pp. 61-65.

${ }^{27}$ R. T. Tung, Phys. Rev. B 45, 13509 (1992).

${ }^{28}$ S. Hardikar, M. Hudait, P. Modak, S. Krupanidhi, and N. Padha, Appl. Phys. A, Mater. Sci. Process. 68, 49-55 (1999).

${ }^{29}$ P. G. McCafferty, A. Sellai, P. Dawson, and H. Elabd, Solid State Electron. 39, 583-592 (1996)

${ }^{30}$ M. Dutta, F. A. M. Koeck, R. Hathwar, S. M. Goodnick, R. J. Nemanich, and S. Chowdhury, "Demonstration of Diamond Based Schottky PIN Diode with Blocking Voltage $>500$ V," IEEE Electron Device Letters (unpublished) 